\title{
Wireless Technology for Pervasive Healthcare
}

\author{
Giancarlo Fortino • Xu Li • Xiaodong Lin • \\ Oscar Mayora • Enrico Natalizio • Mehmet Rasit Yuce
}

Published online: 6 October 2013

(C) Springer Science+Business Media New York 2013

\section{Guest Editorial}

Each human being is unique. This requires medical decisions, practices, and products being tailored to the individual patient. To allow good medical practices, it is necessary to know the history and the genetic predisposition of individuals, as well as their current lifestyle and daily routine. In some cases, the data needed to assess the good functioning and evaluate the performance of a human body can be even larger, e.g. with elderly people, children, athletes, disabled people and people who had health problems in the past. Personalized medicine demands an increased focus on preparing pervasive healthcare systems and applications deployed around users and capable of remotely caring for them and improving their health and well-being anywhere anytime. These systems and applications require a sophisticated integration of microcircuit, medical sensing, and wireless and mobile technologies. They largely benefit users by decreasing the dependability on special

\section{G. Fortino}

Department of Informatics, Modeling, Electronics and Systems (DIMES), University of Calabria (Unical), Rende, Italy

\section{Li}

Huawei Technologies Canada, Ontario, Canada

\section{Lin}

Faculty of Business and Information Technology, University of Ontario Institute of Technology, Ontario, Canada

O. Mayora

CREATE-NET, Trento, Italy

E. Natalizio $(\bowtie)$

Université de Technologie de Compiègne, Rue Roger Couttolenc, 60200 Compiègne, France

e-mail: enrico.natalizio@hds.utc.fr

\section{R. Yuce}

Department of Electrical and Computer Systems Engineering, Monash University, Melbourne, Australia caregivers and eventually reducing healthcare expenses through a more efficient use of healthcare resources and an earlier detection of life-threatening emergency situations. However, the realization of pervasive healthcare sets some additional critical requirements, e.g. realtimeness and faulttolerance, and reliability, security and efficiency challenges compared with traditional hospital-based systems. Exploitation of Information and Communications Technologies as well as Networking and Sensors Technologies will enable cost-effective and efficient healthcare delivery, capable to deal with physical and logical mobility of patients and devices.

This special issue consists of six papers. Each submission underwent three rounds of review with at least three reviewers per round. The selected papers cover different aspects of wireless pervasive healthcare systems along which this special issue has been organized, and range from modeling efforts of the physical phenomena behind the intra/extra body communications to smartphone health applications based on community interactions, including intelligent data collection techniques based on the sensors embedded in the mobile devices as well as transport approaches and systems for dispatching the collected data.

The first paper entitled "Channel and Error Modeling for Wireless Body Area Networks" by Saeed Rashwand and Jelena Misic investigates the channel models and error performance formalization for Wireless Body Area Networks (WBANs). In the first phase of this work, the authors study channel fading models for WBANs. In the second phase, they survey the models which calculate the error performance metrics in WBANs. Then, they select most appropriate error models to design and develop the error performance evaluation models for IEEE 802.15.6-based WBANs and show how to integrate them with the error model in Medium Access Control (MAC). Finally, they discuss integrated PHY and MAC error performance in WBANs.

The second paper "Smart Collaborative Mobile System for Taking Care of Disabled and Elderly People" by Sandra 
Sendra, Emilio Granell, Jaime Lloret, Joel J. P. C. Rodrigues proposes a smart collaborative system based on the sensors embedded in mobile devices. The system determines if a person in a group that wears such devices has suffered an incident or has a problem by looking into the group's activity. A reinforcement learning technique is used to ensure its accuracy when predicting an alarm before it happens and reduce false positives. The authors provide some measurements showing the decisions taken for several common cases and also show the performance of the system when there is a medium size group of disabled or elderly people through simulation.

In the third paper entitled "Energy-Efficient Motion Related Activity Recognition on Mobile Devices for Pervasive Healthcare", Yunji Liang, Xingshe Zhou, Zhiwen Yu, and Bin Guo present a method to recognize physical activity of humans carrying a mobile phone while paying attention to energy efficiency issues such as battery consumption due to signal acquisition and computation workload. In their work they propose a method to recognize physical activity based on the use of a single tri-axial accelerometer tuned with a low sampling frequency for the long-term activity monitoring in addition to an algorithm supporting hierarchical recognition scheme reducing the usage of time-consuming frequencydomain features and adjusting the size of sliding window. The main results of this paper claim effective energy expenditure saves achieved while using the proposed method by extending the battery time on activity sensing and recognition tasks.

The fourth paper "RCare: Extending Secure Health Care to Rural Area Using VANETs" by Mrinmoy Barua, Xiaohui Liang, Rongxing Lu, Xuemin (Sherman) Shen proposes an incentive based delay tolerant long-term health care scheme, which is capable of monitoring patient's health status in a rural area. It uses WBAN, WiFi, and VANETs technologies to provide secure and fair data communication. Vehicles are envisioned to be used in order to store, carry, and forward the personal health information to the health-service-provider located mostly at the city area following an opportunistic routing. Cooperative vehicles are awarded with incentives and good reputation so to guarantee fairness, make the whole system improve network performance and encompass identity based cryptography to ensure security and privacy during the routing period by using short digital signature and pseudoidentity. The evaluation of the scheme shows that the desired level of security is attained while efficiently providing health care to patients who reside in rural areas.

In the fifth paper "Hide-n-Sense: preserving privacy efficiently in wireless mHealth", Shrirang Mare, Jacob Sorber, Minho Shin, Cory T. Cornelius, and David Kotz propose a sensing protocol called Hide-n-Sense (HnS) specific to mHealth network applications that enhances security and privacy features at the link layer, mainly for the data communication between the sensors and a mobile phone. It provides these features with low energy overhead at sensor nodes. In mHealth applications it is very important to preserve patient privacy and maintain data integrity. The work presents three unique techniques to achieve these important requirements with energy efficient communication. It uses an adaptive security technique that dynamically modifies transmission overhead. In addition, it utilizes a MAC striping method and an asymmetric resource requirement to make the protocol strong in terms of security and privacy properties. A prototype system has been implemented to experimentally verify the feasibility of the proposed $\mathrm{HnS}$ protocol.

$\mathrm{Mu}$ Lin et al., in the last paper "BeWell+: Multi-dimensional Wellbeing Monitoring with Community-guided User Feedback and Energy Optimization" propose a mobile application named BeWell+, for monitoring users' behavior along three different health dimensions: sleeping, physical activity and social interaction. BeWell+ automatically infers behavioral patterns using sensors embedded in smartphones, introducing novel mechanisms for an effective user behavior monitoring, namely community adaptive feedback and wellbeing adaptive energy allocation. The system has been evaluated with a 27 person, 19 day field trial. The obtained results indicate that BeWell + operates successfully on consumer-grade smartphones, but users understand feedback and respond by taking steps towards leading healthier lifestyles. Notably, the deployment allowed investigating fundamental issues that may influence the design of future generations of wellbeing mobile apps.

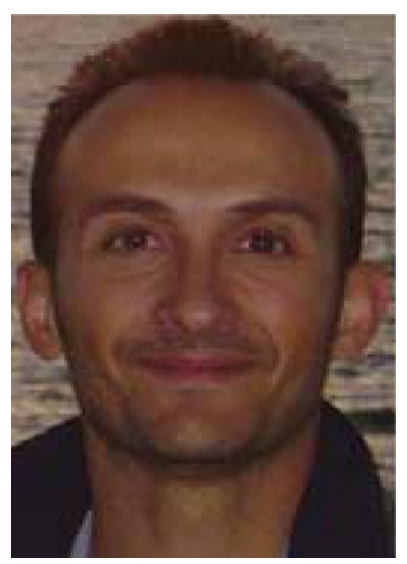

Dr. Giancarlo Fortino received his Laurea (B.S. and M.S.) degree in Computer Engineering from University of Calabria, Italy in 1995, and Ph. D. degree in Computer Engineering from University of Calabria, Italy in 2000 . He is currently Associate Professor of Computer Engineering (since 2006) at the Dept. of Informatics, Modeling, Electronics and Systems (DIMES) of the University of Calabria (Unical), Rende (CS), Italy. He has been a visiting researcher at the International Computer Science Institute, Berkeley (CA), USA, in 1997 and 1999, and visiting professor at Queensland Univ. of Technology, Brisbane, Australia, in 2009. He was nominated Guest Professor in Computer Engineering of Wuhan Univ. of Technology (WUT) on April, 182012. His research interests include distributed computing, wireless sensor networks, software agents, cloud computing, multimedia networks. 


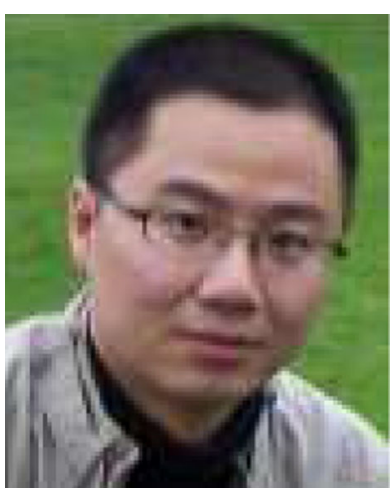

Dr. $\mathbf{X u} \mathbf{L i}$ is a research engineer at Huawei Technologies Canada. Prior to joining Huawei, he worked at Inria, France as research scientist. He received a $\mathrm{PhD}$ (2008) degree from Carleton University, an MSc (2005) degree from the University of Ottawa, and a BSc (1998) degree from Jilin University, China, all in computer science. His current research interests are focused in next-generation wireless networks, along with over 70 refereed publications. $\mathrm{He}$ is an associate editor for the IEEE Transactions on Parallel and Distributed Systems and serving as TPC co-chair for GC'13 Ad Hoc and Sensor Networking Symposium.

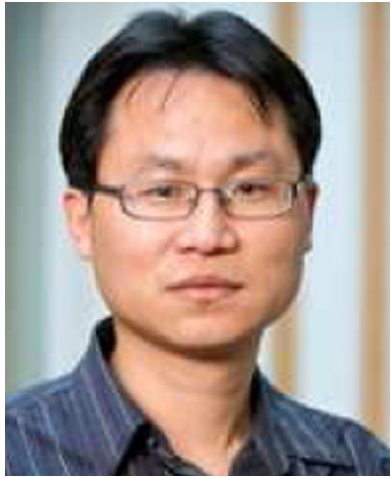

Dr. Xiaodong Lin received the $\mathrm{Ph} . \mathrm{D}$. degree in information engineering from Beijing University of Posts and Telecommunications, Beijing, China, and the Ph.D. degree in electrical and computer engineering from the University of Waterloo, Waterloo, ON, Canada. $\mathrm{He}$ is currently an Associate Professor of Information Security with the Faculty of Business and Information Technology, University of Ontario Institute of Technology, Canada. His research interests include wireless communications and network security, computer forensics, software security, and applied cryptography. He is a Senior Member of the IEEE.

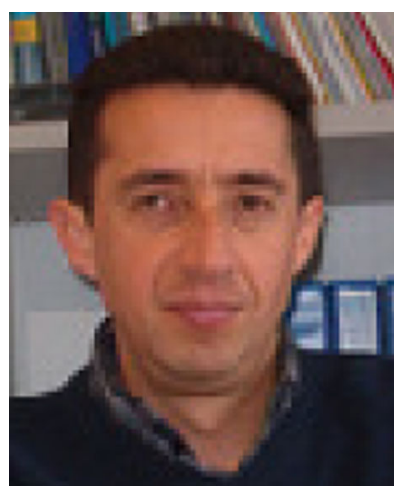

Dr. Oscar Mayora is head of the Mobile and Ubiquitous Technologies Group in CREATE-NET. He is a Senior Member of the ACM and SIG-CHI, and is a former President of ACM SIG-CHI for Mexico. He is the founder and a permanent member of the Steering Committee of the Pervasive Health Conference. He has extensive experience participating and leading projects in the field of mobile technologies applied to healthcare.

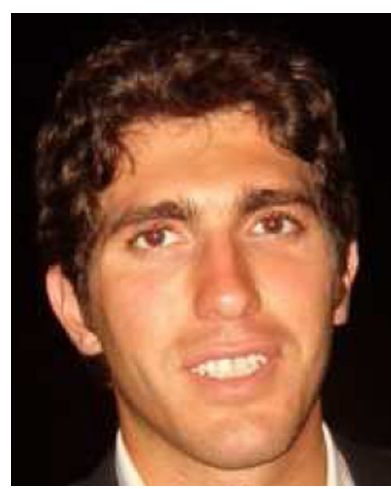

Dr. Enrico Natalizio is an Assistant Professor at the Université de Technologie de Compiègne, in the Network\&Optimization group within the Heudiasyc Lab. He obtained his $\mathrm{Ph}$. D. from the Università della Calabria, Italy and he was a visiting researcher at the BWN (Broadband Wireless Networking) Lab at Georgia Tech in Atlanta, GA, USA. From December 2005 until September 2010, he worked as a research fellow and a contract professor at the Università della Calabria, Italy. From October 2010 to August 2012 he worked with the FUN Team at INRIA Lille - Nord Europe as a postdoctoral researcher. His current research interests include group communication in wireless robot and sensor networks and coordination and cooperation among swarm networked devices.

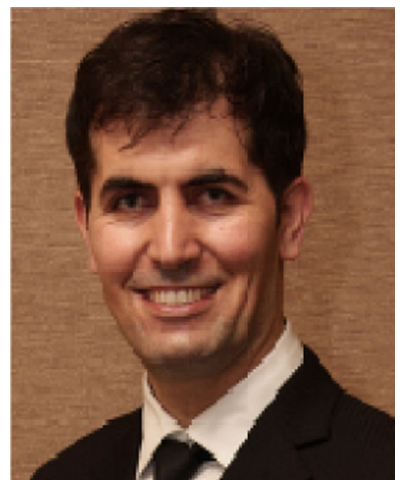

Dr. Mehmet Rasit Yuce received the M.S. degree in Electrical and Computer Engineering from the University of Florida, Gainesville, Florida in 2001, and the Ph.D. degree in Electrical and Computer Engineering from North Carolina State University (NCSU), Raleigh, $\mathrm{NC}$ in December 2004. He was an academic member at the School of Electrical Engineering and Computer Science, University of Newcastle, Australia until Jul 2011. In July 2011, he joined the Department of Electrical and Computer Systems Engineering, Monash University, Melbourne, Australia. His research interests include wireless implantable telemetry, wireless body area network (WBAN), bio-sensors, integrated circuit technology dealing with digital, analog and radio frequency circuit designs for wireless, biomedical, and RF applications. 